\title{
A Profiling Architecture Based on Web for Supporting Configuration Management in Actuator Networks
}

\author{
In-Cheol Hwang and Do-Hyeun Kim \\ Department of Computer Engineering, Jeju National Univ. \\ spfe7388@hanmail.net, kimdh@jejunu.ac.kr
}

\begin{abstract}
Recently, IoT(Internet of Things) applications based on actuator networks not only need an appropriate way for representing information about actuator's characteristics but also a common information for managing the involved elements. This paper presents the profiling architecture based on Web services for supporting configuration management in actuator networks. We design and implement a profile management tool based on XML (Extensible Markup Language) for representing actuator descriptions as well as the flow chart for generating and editing the actuator configuration profile in actuator networks.
\end{abstract}

Keywords: Profiling framework, Actuator networks, Actuator description

\section{Introduction}

There is a growing demand in managing flexible devices that aims to interoperate among heterogeneous sensors, actuators and other services. Sensor networks have enabled a range of applications where the objective is to observe an environment and collect information about the observed phenomena or events. Sensors gather information about the physical world, while actuators make decisions and perform actions that affect the environment [1]. Actuators are able to change parameters in the environment as well as the intrinsic properties. The actuator networks perform the appropriate action according to the context and growing interest. The properties of actuator networks are more complex than the existing IT systems. The autonomous command based on context performed different functionality, like coordination with sensor networks, connectivity with server and internet, and the required response of the executed results. It is the hardware properties of various devices to coordinate with the existing industrial systems. In addition, the interaction between actuator and sensor is necessary because the elements of actuator networks must operate autonomously. In order to manage actuator networks it needs to identify actuators and grasp properties. The application of actuator networks in various ubiquitous application services needs not only the distinction of actuators but also, the information for detailed abilities and chat eristic of actuators.

Profile is a set of information for particular works. Various services based on many existing profile information are supplied suitably to users. In order to provide services in ubiquitous applications and sensor networks, the profile of the actuator network is required to manage various heterogeneous actuators $[1,2]$.

This paper proposes the profiling architecture for providing configuration management services and managing devices in actuator networks. We develop a profile management tool based on XML to represent actuator descriptions as well as the flow chart for generating the actuator configuration profile in actuator networks. The architecture and tool provide a way 
to configure devices in a user-friendly and expressive way thus enabling effective semantic description of the devices.

The paper is organized as follows. In the next section, we discuss the related works. Section 3 deals with the proposed profiling framework for configuration of actuator. In Section 4 our implementation of register module for actuator profile support and certify support techniques with the programming language C\# will be described. An overall conclusion and future work is summarized in Section 5.

\section{Related work}

The objective of developing profiling architecture is to support the users in the intelligent environment such that they can access any device in a seamless fashion. A profiling architecture should effective included newly evolved devices and/or enable modifications to existing devices with minimal effort [3].

The management framework has some weaknesses especially related to extensibility configuration management and efficient application development processes. Today, researchers and developers in the network and system management area have begun to investigate the use of XML technologies seems to promise a fancy way out of this trouble. These have been applying XML technologies to developing XML-based management systems to solve the problems that exist in the network management [4]. A Network Management System proposes one kind model of MIB transform to XML view. It also proposes a common network management system and gives examples of specific applications. Technology of the system can be used as network management and software system development reference [5].

These technologies provide the option to interconnect heterogeneous devices and services; however, not all of them can equally accommodate the others under the same hood. One of the very promising technologies that can be used to address this issue is the Devices Profile for Web Services (DPWS) [6], which can work with these technologies and provide interoperability among the different devices and services in a smart home environment. The Devices Profile for Web Services (DPWS) was developed to enable secure Web service capabilities on resource-constraint devices. It allows sending secure messages to and from Web services, dynamically discovering a Web service, describing a Web service, subscribing to, and receiving events from a Web service. DPWS can be used for machine to machine communication whereas a specific client uses a specific service hosted on a device. DPWS is not the first SOA that targets device-to-device communication. Technologies such as Open Service Gateway Initiative (OSGi), Home Audio/Video Interoperability (HAVi), Java Intelligent Network Infrastructure (JINI) and Universal Plug and Play (UPnP) are similar approaches.

The IEC 62390 standard Device Profile Guideline precise the scope of the device profiles in the sense of this paper in focusing on the networked automation devices also known as networked embedded devices or system [8]. In principle there are three device models representing the device parameters and behavior. These are the parameter list model (an ordered or unstructured list of parameters possibly with related constraints and behavior among the parameters), the well-known function block model (see standards like IEC 611313, IEC 61804-2 and IEC 61499) and the object models (equivalent to the software programming paradigm object orientation). IEC 62390 uses Functional Elements (FE) as an abstract term covering all three models [7].

The Device Configuration Management Tool (DCMT) is placed to the context of the device configuration management framework which conceptualizes how to model the field devices, to enable a way to access the context of the devices, and to provide a graphical 
presentation of the system for a user in accordance to his/her role and preferences. This tool aims to contribute to context management in an automated system which supports decision making of a user in complex environments via designed human-machine interface in accordance to user role, intentions, state and situational context. The designed DCMT generates configuration files of each device, to enable semantic representation of the field level. The configuration of the devices is done by an installer of the system. For this reason a graphical user interface was developed, which provides a smooth device configuration to the installer, regardless of his or hers engineering background, especially in the field of ontology [8].

\section{Profiling Framework for Configuration of Actuator}

The elements that are required to support all node composition profile of actuator networks are actuator register, edition, and storing, etc. Profile management and registration schematics for these components to support actuator profile, are presented. By means of actuator register, edition and management module, the profile for the actuator registration and management is stored in XML format and the profile is stored in the database for redundancy. XML parser reads XML data from the XML-based profile to support actuator composition profile. It also support edition and registration of XML based profile. Profiles read only the XML data from the XML-based profile parser. The XML editing module supports to add, to modify, and to delete a profile of the actuator and also create data connection to connect to the database and store data in database. Figure 1 shows the profiling framework of the system to support the actuator composition profile.

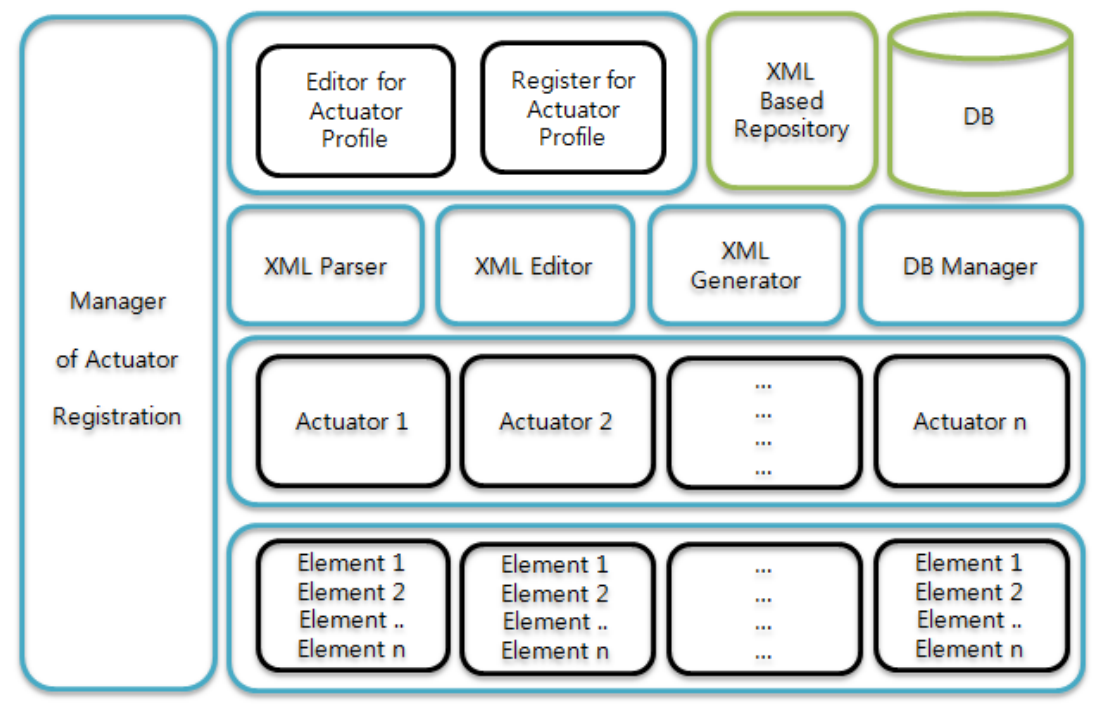

Figure 1. A profiling framework of actuator supporting profile

Firstly, it is decided whether recall the file that contains the actuator information or provides a new file. If we select a new file, it is decided actuator node type, manufacturing-related information, the location address information, mobility, connection information and operational status. If we read the existing file, it can edit actuator information by selecting a node number that corresponds to the actuator or it 
can modify, store the information in the database and in XML file. Figure 2 shows the flowchart of actuator register for supporting profile.

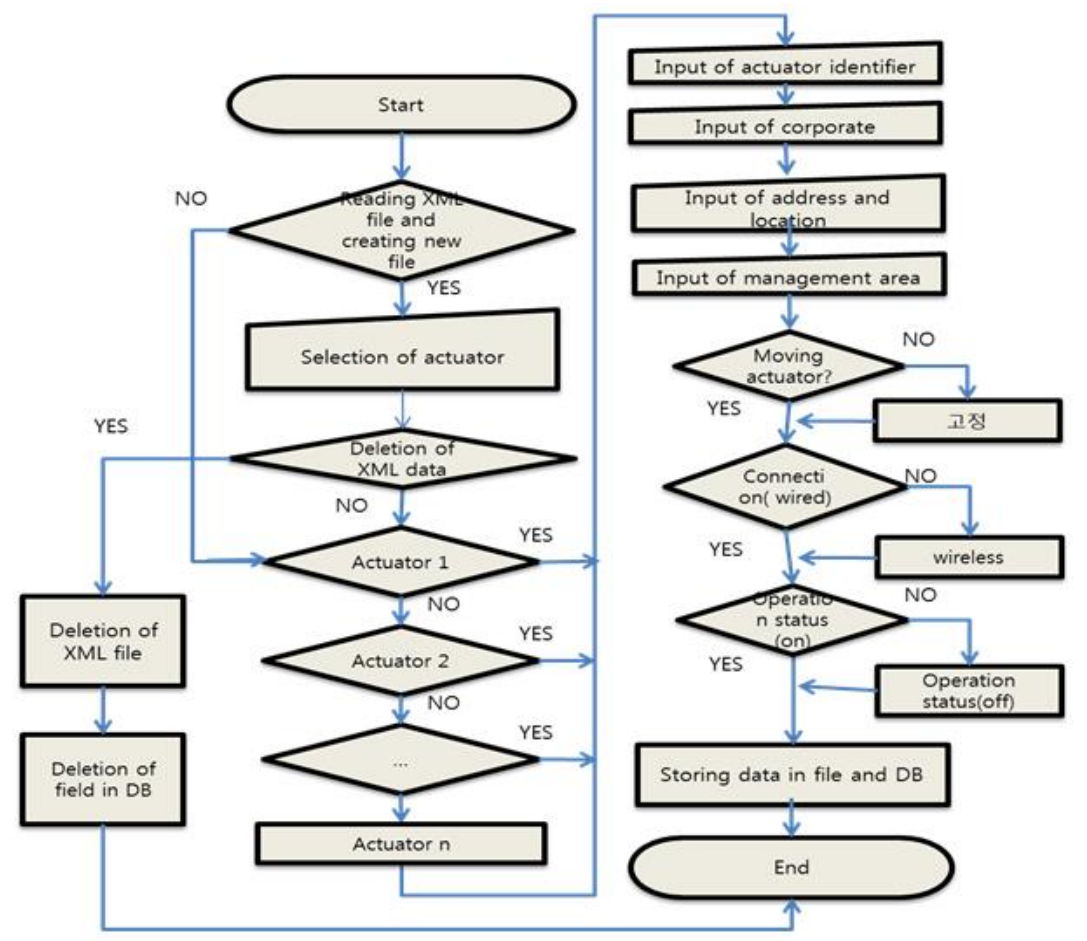

Figure 2. Supporting process for actuator composition profile

Table 1 shows each specific function. 'XML parser function' is used to If user select the XML data in the XML document and to import the data to the actuators; if we want to add, modify and delete actuator related information, 'XML editing function' can be used in the form of XML; to make a new XML document, 'XML supporting function for database connection 'DB connection function' is provided; and to save the change data, 'DB edition function' can be used.

We can see here how actuator registration page behaves. First, the user can choose one of the two options. Load profile option provides the direct input using the screen and from file using XML file format. If we choose the direct input, we have to put the information on the screen one by one. If we choose uploading the files that are not direct input, select simply the number of nodes that corresponds to the number of the actuator information, then the information will be shown on the screen. User can modify and delete the contents on the screen whenever he wants. The two options provided can work at the same time to save the entered information. 
Table 1. Details function for actuator registration and management

\begin{tabular}{|c|l|}
\hline Element & \multicolumn{1}{|c|}{ Description } \\
\hline XML Parser & Analysis of Actuator.Xml and Extraction of information \\
\hline XML Editor & Addition, update, deletion of xml data \\
\hline XML Generator & Creation of new XML data \\
\hline DB Connecter & DB connection for reading and storing data \\
\hline DB Editor & Addition, update, deletion of DB contents \\
\hline
\end{tabular}

\section{Implementation and Results based on Web}

In order to support the actuator node profile, ASP.NET technology based on C \# by Microsoft Visual Studio 2008 is used and web-based implementation are done.

Figure 2 is a web page screen of the actuator registration and management. Actuator registration page provides two kinds of information's, basic information and hardware information. Here visualization based services are provided to show the profile information to the user. The basic information part in the actuator registration shows adapter name in connected drive, service area, the IP address, port number, transmission method and data transmission interval, etc. The hardware information part provides the function of addition, modification, and deletion.

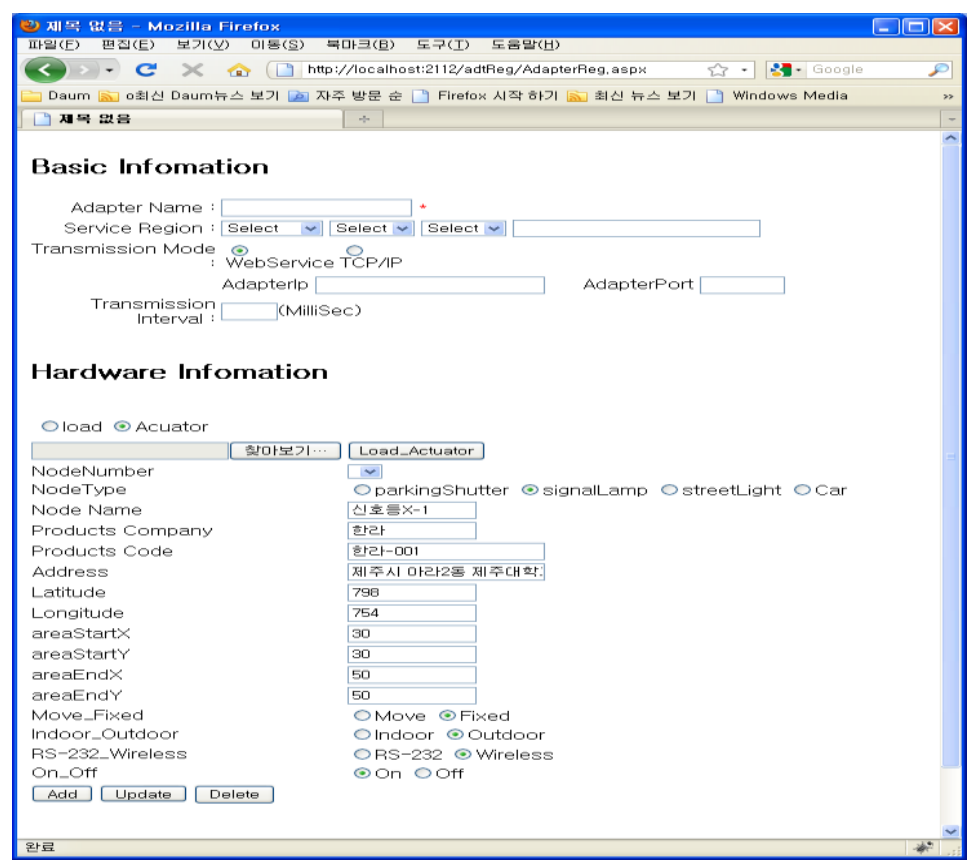

Figure 3. Actuator registration screen

Figure 3 shows the registration and profile information screen. It shows the profile in the form of XML which is generated by providing actuator information. The actuator file that is 
to be input must contain the information regarding node type, node name and manufacturer, etc. If we click on the add button, the actuator profile information will be generated in the form of XML format and this information will be stored in the database.

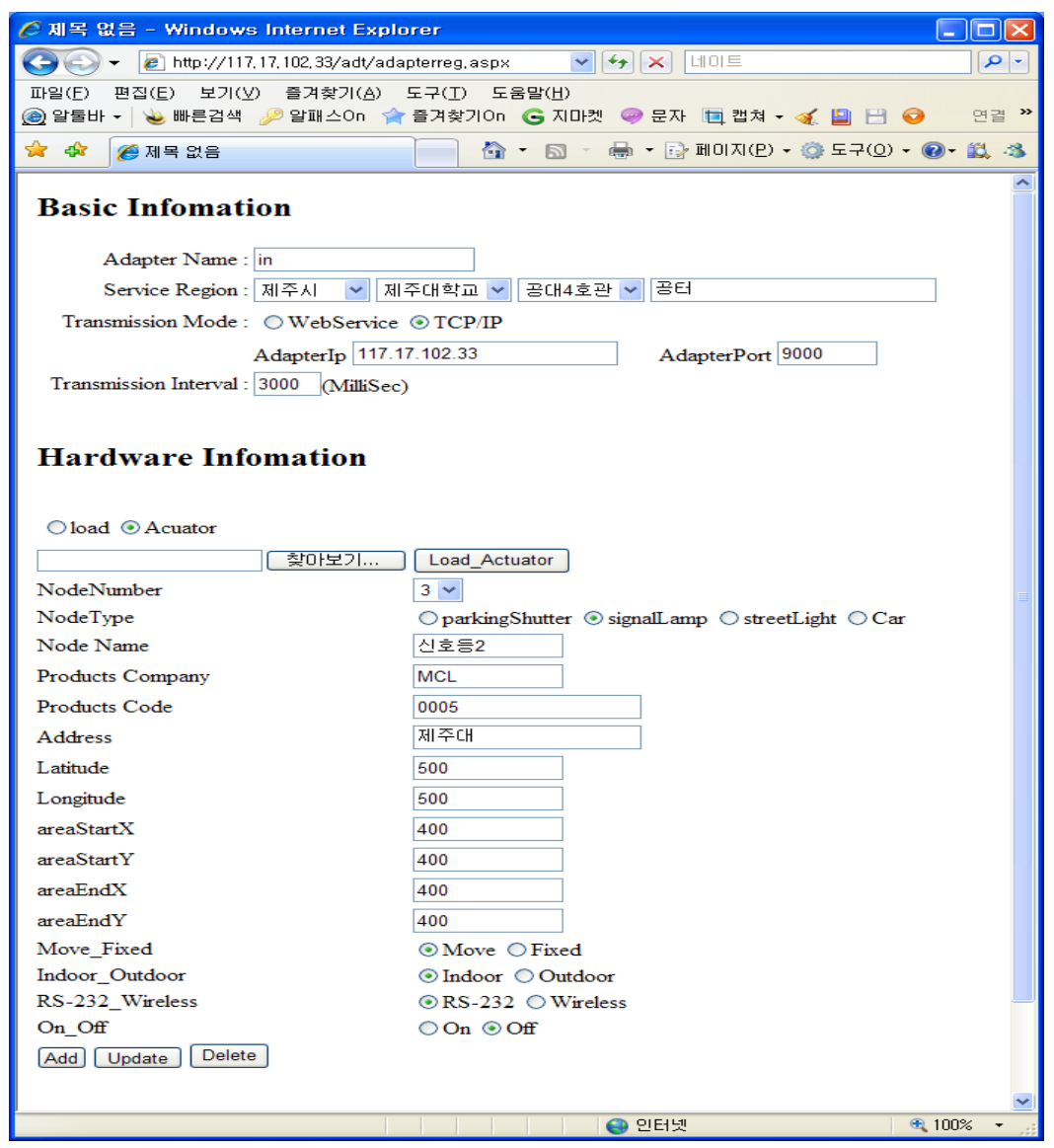

Figure 4. Actuator profile results screen

Figure 4 shows the results screen. It displayed the actuator profile information of the selected node number; the selected nod number in this case is 3 . The displayed information of node 3 is node type, node name, manufacturer, product code, address, latitude, longitude, mobility, and indoor or outdoor, communication system, and the operation status.

In addition, we can read, modify, and delete actuator profile by using the node number of the actuator. Similarly, actuator registration and management function for reading the profile in the form of XML (Load_A_spec_click ()), ) additional functions (AddXMLElement (), ) modification function (ModifyXMLNode (), delete function (bt_Delete_Click ()) are also provided. Other functionality we provided to the users is to edit the actuator information, store the profile information in the form of XML (SaveXMLFile ()), and in the database (ActuatorDBConnnet ()).

Figure 4 shows the setting environment to read the profiles of the XML type that involves the stored actuator information. The path is set here. After setting the path, when we click on the Load Actuator button, the information related to the actuator profile will be displayed in the form of XML. Here the user can select the node number that corresponds to the actuator information. 


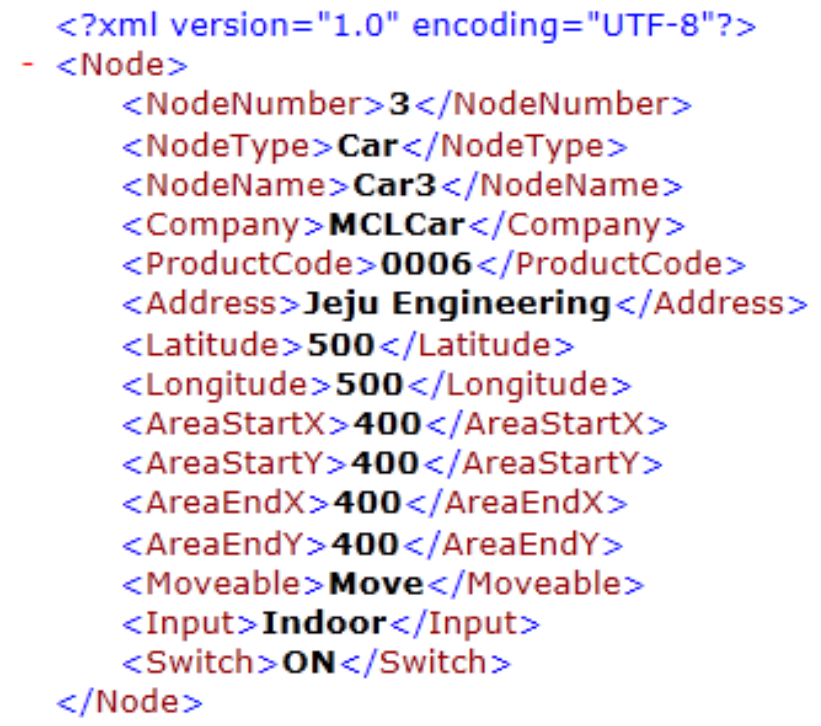

Figure 5. An example for configuration profile of the car actuator

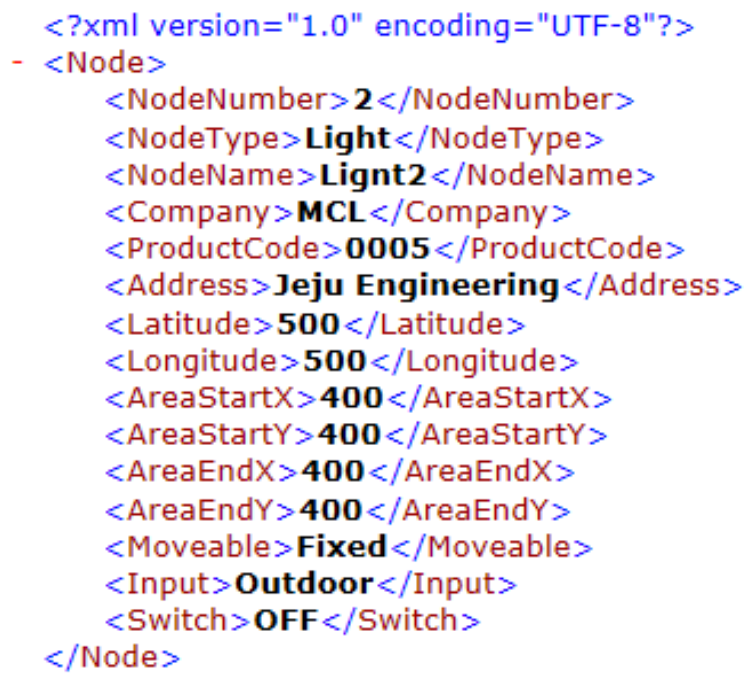

Figure 6. An example of configuration profile for a traffic light

As mentioned earlier, depending on the process of actuator configuration profile supporting, first make a file in XML format. For example, node number increases by 1, if a car is registered to a new actuator. The node type indicates the type of the actuator, where set a particular kind of actuator. Specify a node name by the user, manufacturing company, product number, address, latitude and longitude. The extent of actuator management represents by AreaStartX, AreaStartY, AreaEndX, and AreaEndY. The actuator mobility (Moveable) represents the fixed and mobile. Space represents indoor or outdoor display. Connection or interface shows the wireless LAN or RS-232. The current operating state represents on or off. Figure 5 shows an example of the actuator composition profile for a car, and Figure 6 shows the actuator composition profile for a traffic light. 


\section{Conclusion and Future Work}

Recently, a movement from actuators controlled by users to automatic, autonomous and self-configuring actuators is noticeable. Profiles define which configuration management is used, how they are adapted and in which way they are used to achieve a certain aim. Thus, profiles are meaningful information for achieving interoperability between devices implementations of different vendors. This paper presents the profile framework for providing ubiquitous services and hardware management in actuator networks. We design and implement a profile management tool based on XML to represent actuator descriptions and the flow chart for generating and editing the actuator configuration profile in actuator networks. We will further investigate how device templates can improve profile management tool and complete the toolkit. For implementing configuration management on devices with even lower resource constraints and for solving problems with investigated variable actuators in future research.

\section{Acknowledgements}

This work was supported by ETRI(Korea) [Development of USN/WoT Convergence Platform for Internet of Reality Service Provision (13ZC1130)]. "This work was supported by the National Research Foundation of Korea(NRF) grant funded by the Korea government(MEST) (No. 2011-0015009)."Corresponding author; DoHyeun Kim (e-mail: kimdh@jejunu.ac.kr).

\section{References}

[1] S. X. Yang and Y. N. Zuo, "Adaptive profiling framework and system for service provisioning”, International Conference on E-Commerce, (2003) June 24-27; California, USA.

[2] J. Indulska and R. Robinson, "Experiences in Using CC/PP in Context-Aware Systems", Springer, Lecture Notes In Computer Science, vol. 2574, (2003).

[3] J. Parra, M. A. Hossain, A. Uribarren, E. Jacob and A. E. Saddik, "Flexible Smart Home Architecture using Device Profile for Web Services: a Peer-to-Peer Approach", International Journal of Smart Home, SERSC, vol. 3, no. 3, (2009).

[4] J. M. -k. Guo, Y. -m. Yu, M. Wang and Q. Yu, "Research and Implementation of Network Management System Based on XML View", Proceedings of 2010 International Conference on Logistics Engineering and Intelligent Transportation Systems (LEITS), (2010) November 26-28, Wuhan, China.

[5] F. Zheng and Z. Cui, "The New Way of Configuration Management of Network Devices under Mutable Environment", Proceedings of the 3rd International Symposium on Knowledge Acquisition and Modeling (KAM), (2010) October 20-21; Wuhan, China.

[6] E. Zeeb, A. Bobek, H. Bohn and F. Golatowski, "Service-Oriented Architectures for Embedded Systems Using Devices Profile for Web Services", Proceedings of 21st International Conference on the Advanced Information Networking and Applications Workshops, AINAW '07, (2007) May21-23, Ontario, Canada.

[7] C. Diedrich, M. Muhlhause, M. Riedl and T. Bangemann, "Mapping of smart field device profiles to web services", IEEE International Workshop on Factory Communication Systems WFCS, (2008) May21-23, Dresden, Germany.

[8] A. Kuutti, A. Dvoryanchikova, A. Lobov, J. L. M. Lastra and T. Vantera, "A Device Configuration Management Tool for Context-Aware System", Proceedings IEEE International Conference on Industrial Informatics (INDIN), (2012) July 25-27, Beijing, China.

[9] Y. H. Zhang, Q. Y. Dai and R. Y. Zhong, "An Extensible Event-Driven Manufacturing Management with Complex Event Processing Approach”, International Journal of Control and Automation, SERSC, vol. 2, no. 4, (2009). 


\section{Authors}

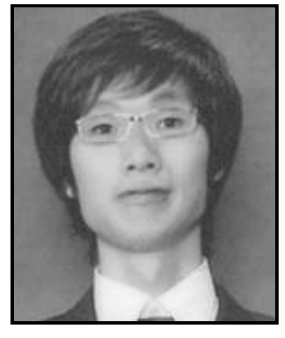

\section{In-Cheol Hwang}

He received the B.S. degrees in Computer Engineering from Jeju National University, Jeju, Korea, in 2011 respectively. He joined the Commers Corporate, Korea, in 2010. Since 2010, he is currently a researcher at the Department of Computer Program Development at Cermers Corporate, Korea. His research interests include Web programming, Sensor networks.

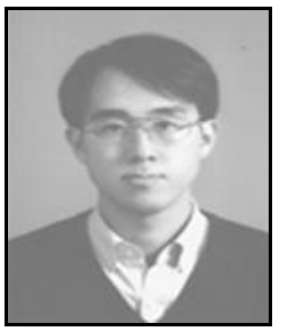

\section{Do-Hyeun Kim}

He received the B.S., M.S. and P.D degrees in Electronics Engineering from Kyungpook National University, Taegu, Korea, in 1988 and 1990, 2000 respectively. He joined the Agency of Defense Development (ADD), Korea, in 1990. Since 2004, he is currently a professor at the Department of Computer Engineering at Jeju National University, Korea. His research interests include sensor web, optimization algorithm and context prediction. 
International Journal of Control and Automation Vol.6, No.5 (2013) 